\title{
Comparative Analysis of Feeding Techniques for Micro strip Patch Antenna and Smart Antenna Applications in Mobile Communication
}

\author{
Priti Sharma ${ }^{1}$ and Dr. R. L. Yadava ${ }^{2}$ \\ ${ }^{1}$ Research Scholar Mewar University Rajasthan, Assistant Prof.BIT Noida \\ ${ }^{2}$ Dept.of Electronics \& Comm., Enggs, Galgotia College of Engineering Greater Noida,
}

\begin{abstract}
This paper describes two folds. First it describes comparative analysis of different feeding techniques for microstrip patch antenna wireless applications. Where it covers two broad categories of feeding approachcontracting (coaxial and microstrip feeds) and non-contracting (aperture coupling and proximity coupling feeds). Today as smart antenna has emerged as an auspicious technique to improve the performance of cellular mobile system. In second section authors propose a MIMO structure, consist of multiple antenna elements and its application in mobile communication with the key characteristics features, benefits, drawbacks, types of smart antennas and their working mechanism..
\end{abstract}

Key Words: Microstrip Antenna (MSA), Feeding Techniques, Smart Antenna.

\section{Introduction}

A microstrip antenna with its features such as low profile, light weight, easy fabrication makes it very useful in cellular communication. Feed line is used in microstrip antenna for excitation to radiate wave by direct or indirect contract. Microstrip antennas are fed by two ways -1. Contracting, (coaxial and microstrip feeds), 2 . Non contracting (aperture and proximity coupling feeds). The WiMAX technology is a widely growing field in recent mobile communications with its application in mobile devices such as smart phones and hand held computers. This gives user the mobility to move with in a broad coverage area with a wide range of network connection. Along with the mobile users, home users also find wireless communication better as it gives location freedom and ease of installation.

Mobile communication requires small, low-cost, low profile antennas which has Omni directional radiation pattern in horizontal planes. Microstrip patch antenna meets all requirements and various types of microstrip antennas have been designed for use in mobile communication systems.

\section{Feeding Techniques}

The feed mechanism plays an important role in the design of microstrip patch antennas. In the contacting feed method, the RF power is directly fed to the radiating patch with the help of a connecting element such as a microstrip line. In the non-contacting scheme energy is transfer between the microstrip line and the radiating patch electromagnetic field and coupling is done. That is a microstrip patch antenna can be fed either by coaxial probe or by an inset microstrip line. Coaxial probe feeding is sometimes advantageous for applications like active antennas, while microstrip line feeding is suitable for developing high-gain microstrip array antennas. In both cases, the probe position or the inset length determines the input impedance.

\subsection{Microstrip Line Feeding}

In Microstrip line feed the edge of the microstrip patch is directly connected to a conducting strip. The width of strip is smaller than the patch so the feed can be etched on the same substrate to provide a planar structure. It is simple to model and easy to match by controlling the inset position. Fig.1 shows the microstrip line feeding for a rectangular patch antenna.

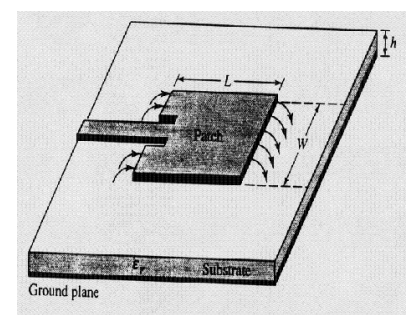

Fig.1 Microstrip line Feeding 
However the disadvantage of this method is that as substrate thickness increases, surface wave and spurious feed radiation increases which limits the bandwidth [1-4].

\subsection{Coaxial Feeding}

Coaxial feeding is a method in which the inner conductor of the coaxial is extended and attached to the radiation patch of the antenna while the outer conductor is connected to the ground plane. This type of feeding scheme facilitate that the feed can be placed at any desired location inside the patch in order to match with its input impedance, as shown in Fig. 2.

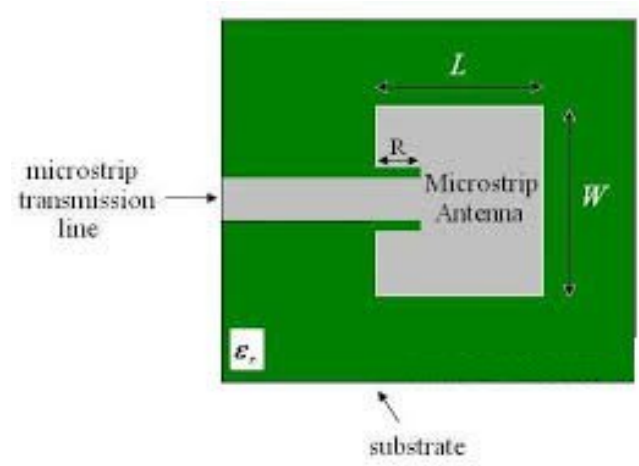

Fig. 2 Coaxial Feeding

This scheme provides the advantages of easy of fabrication, easy to match and Low spurious radiation. On the other hands it suffers from the drawbacks of narrow bandwidth, difficult to model specially for thick substrate and possess inherent asymmetries which generate higher order modes which produce crosspolarization radiation.

\subsection{Aperture Coupling}

In aperture coupling two different substrate the radiating patch and microstrip feed line are separated by the ground plane. The energy of microstrip feed line is coupled to the patch through a slot on the ground plane separating two substrates to make a coupling effective. In this coupling technique the top substrate uses a thick low dielectric constant while the bottom substrate is of high dielectric substrate.

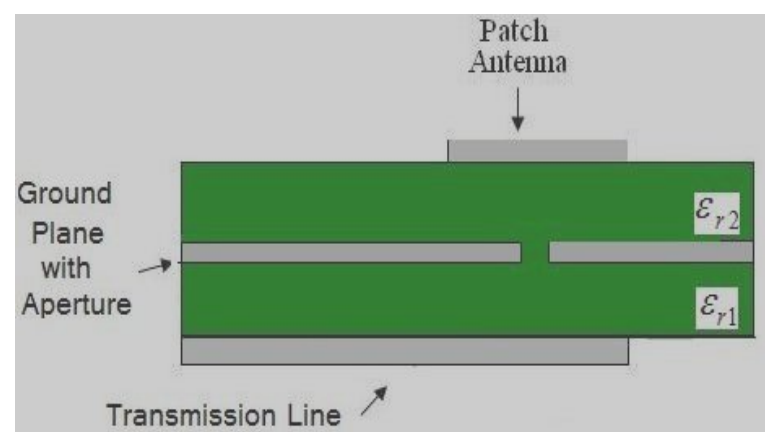

Fig. 3 Aperture Coupled Feeding

In the middle, the ground plane isolates the feed from radiation element and minimizes interference of spurious radiation for pattern formation and polarization purity. Aperture coupling feeding facilitate with the advantages of no physical contact between the feed and radiator, wider bandwidths, and better isolation between antennas and the feed network and independent optimization of the feed mechanism and the radiating element.

\subsection{Proximity Coupling}

Proximity coupling also refereed as electromagnetic coupling feeding in which between two substrates there is a feed line. The radiating patch is on the top of the upper substrate as shown in Fig 4. This scheme has the largest bandwidth, and low spurious radiation. 


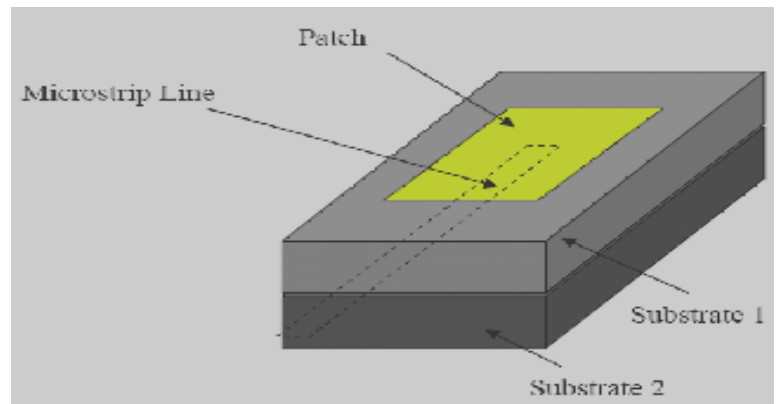

Fig.4 Proximity coupled Feeding

However fabrication is difficult and length of feeding stub and width-to-length ratio of patch is used to control the matching. [5-8]

\section{Antenna System For Wireless Mobile Communication}

Few of the commercial applications which implement microstrip antennas are as follows:

Table 1 Microstrip Antenna Applications [9]

\begin{tabular}{|c|c|c|}
\hline S. No. & Applications & Frequency band \\
\hline 1. & Global Positioning Satellite & $1575 \mathrm{MHz} \& 1227 \mathrm{MHz}$ \\
\hline 2. & GSM & $890-915 \mathrm{MHz} \& 935-960$ \\
& MHz \\
\hline 3. & WLAN & $2.40-2.48 \mathrm{GHz} \& 5.4 \mathrm{GHz}$ \\
\hline 4. & Cellular Video & $28 \mathrm{GHz}$ \\
\hline 5. & Direct Broadcast satellite & $11.7-12.5 \mathrm{GHz}$ \\
\hline 6. & Automatic Toll Collection & $905 \mathrm{MHz} \& 5-6 \mathrm{GHz}$ \\
\hline 7. & Collision Avoidance Radar & $60 \mathrm{GHz}$ \\
\hline
\end{tabular}

\subsection{Antenna Requirements in Recent Wireless Communication Systems}

From traditional wired communication system, the wireless communication system are moving towards higher transmission speed, higher frequency ranges to increase capacity to achieve better quality signal and Signal multiplexing. The antenna requirements for these systems are:

a. High-gain and high-efficiency technology

b. Multi-antenna technology

Smart Antennas are a kind of technology which fulfils these requirements. The latest smart antenna systems can increase the antenna gain even in a mobile system and expands the service coverage area. The designed system capacity can be increased by using multiplexing techniques based on baseband signal processing. Multiantenna systems (antenna arrays) can achieve very high performance with high-level functions corresponding to the wireless system requirements.

\section{Smart Antennas}

Existing digital cellular networks provide a limited capability which through the network boundaries and concept of smart antennas brings in the world of wireless communication. Smart antenna employs the concept of multibeam technology, which improves the performance of FDMA, CDMA and TDMA.

In hierarchical system approach where demand based frequency allocation is required Smart Antennas are best suitable candidate. SA's application in cellular system provides the advantage of reduced inter symbol interference (ISI), decreases co channel interference, better bit error rate, increases receiver sensitivity, reduced power consumption and RF pollution. Best applications of smart antennas are for cognitive radio where flexibility is provided by software radio technology and the greatest advantage of SA is a very high security. 


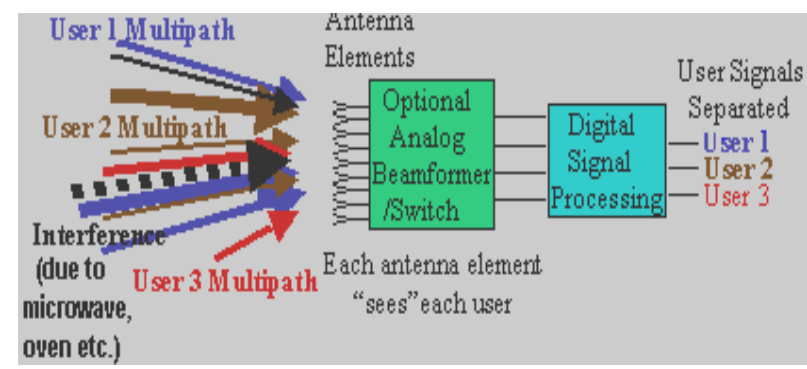

Fig. 5 Smart Antenna System [5]

The main problems with wireless communications are co channel interference which limits the system capacity, inter symbol interference and signal fading due to multipath. Signals are arrived at the receiver through different directions, SA's can exploits the difference between desired signal and co channel interference and thereby reduce the co channel interference Similarly reflected multipath signals are also arrived at receiver from different directions and spatial processing can use these differences to attenuate multipath and thereby reduce ISI and fading. To overcome the drawbacks of omnidirectional antennas Smart Antenna concept comes in front (Fig.5).

Basically a Smart Antenna which is co-located with a base station combines an antenna array with a digital signal processing capability to transmit and receive in an adaptive, spatially sensitive manner. SA's enhances the wireless links capacity through a combination of diversity gain, average and interference suppression. A smart antenna is a system, where an array of antennas direct different transmission/reception beams collectively toward each user in the system. This method of transmission and reception is called beam forming. In beam forming, each user's signal is multiplied with complex weights that adjust the magnitude and phase of the signal to and from each antenna. This causes the output from the array of antennas to form a transmit/receive beam in the desired direction and minimizes the output in other directions [9-10].

\subsection{Types of Smart Antennas}

Smart antennas system is influenced by different technical terms such as SDMA, spatial processing, phased array, adaptive array system, digital beam forming (Fig.6). On the basis of transmit strategy Smart antennas are broadly categorized as:

1. Switched Beam: In this a finite number of fixed pre-defined patterns are transmitted (sectors based)

2. Adaptive Array: This includes an infinite number of patterns that are adjusted in real time can be transmitting (scenario based)

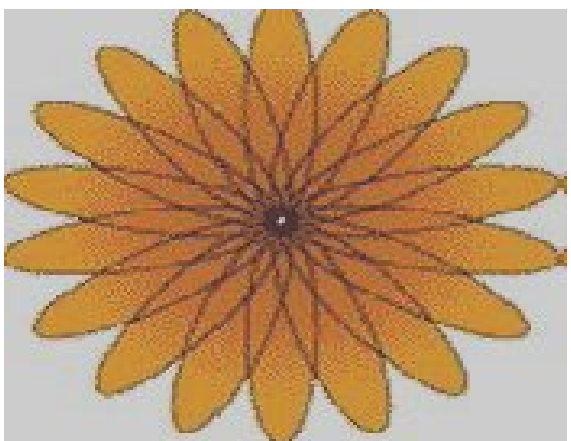

Fig.6. Switched beam system coverage patterns

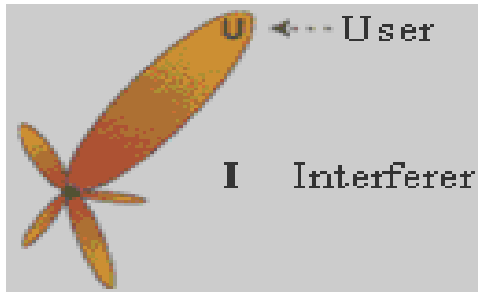

Fig.7 Adaptive Array Antenna 


\subsection{Smart Antenna System Architecture}

\subsubsection{Working of Smart Antenna Systems}

The working concept of smart antenna is based on the assumption that within the range of the desired device no other device or transmitting frequency lies i.e. the interference signals are weaker than the transmitted and received signals. SA's uses the concept of spatial filtering according to which it receives energy from a particular direction, while simultaneously rejecting it from other directions. Spatial filtering makes SA's more effective in the remote areas so the location of the device can be easily traced.

Through internal feedback control system, smart antennas modify the beams of each remote user generated by the base station. Each approach (switched beam, adaptive array system) forms a main lobe towards individual users and attempts to reject interference or noise from outside of the main lobe.

\subsubsection{Uplink Frequency}

Uplink is the communication link from the mobile user to the base station .It is supposed here that a smart antenna is only employed at the base station and not at the handset or subscriber unit. Omnidirectional antennas are implemented with the mobile units and it is the major responsibility of the base station to separates the desired signal from interference using spatially selective reception. This process is termed as uplink frequency. In the process of up linking the signal received from spatially distributed antenna element is multiplied by a weight i.e. a complex value of amplitude and phase further these signals are combined to produce array output.

\subsubsection{Downlink Processing}

Downlink is a communication from base station to mobile unit .Base station utilizes spatially selective transmission while the mobile unit doesn't use spatially selective reception. The process of spatially selective transmission is called as downlink processing.

The communication system uses either frequency division duplex (FDD) or time division duplex(TDD) according to which type of downlink processing is decided. The reason behind this is ,because in most FDD systems the uplink and downlink fading may be considered independent, whereas in TDD systems the uplink and downlink channels can be considered reciprocal [13]. Therefore, in TDD systems uplink channel information may be used to attain spatially selective transmission. In FDD systems, the uplink channel information cannot be used directly and other types of downlink processing have to be considered.[6], [9][14]

\subsubsection{Basic Working Mechanism}

A smart antenna system can perform the following functions:-

(a) Direction of Arrival: First, using the direction of arrival algorithm, the direction of arrival of all the incoming signals, interfering signals and the multipath signals are estimated.

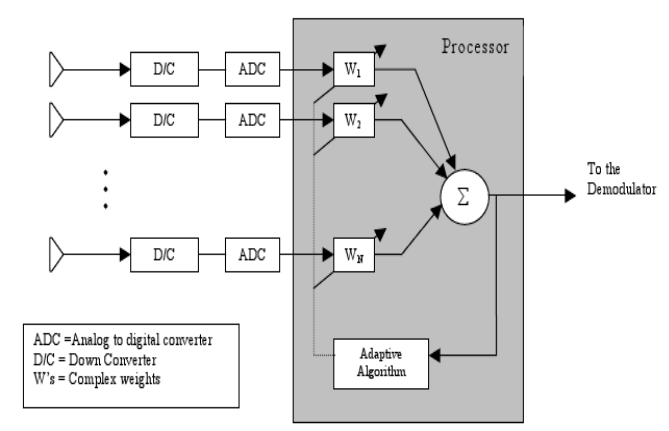

Fig 4: Block diagram of Adaptive array systems

(b) Sorting of desired Signal: Secondly, the desired user unwanted incoming signals.

(c) Lastly, a beam is tipped in the desired signal direction and the user is tracked as he moves while placing voids at interfering signal directions by constantly updating the complex weights. [6]

\section{Limitations Of Smart Antennas}

1. Complex: Fault finding is more complex with SA'a which makes it more complicated than traditional antennas.

2. More Expensive: As Smart antenna uses latest processing technology they are more expensive.

3. Larger Size: As smart antenna is consists of antenna arrays, their size is much larger than traditional antennas. 
4. Location: For optimal operation location of smart antenna is considered. 5.2 Benefits of Smart Antenna Technology

1. Interference rejection: Smart antenna works on the principal of spatial filtering antenna receives energy from a particular direction, while simultaneously rejecting it from other directions. In addition, they also have nulls in their radiation pattern in the direction of other mobile users in the vicinity. Therefore, there is often negligible co-channel interference.

2. Range Improvement. Since, smart antennas is an array of different antenna elements they give rise to narrow beam with increased gain when compared to conventional antennas using the same power. Increase in gain, enhance the range and therefor increase the coverage area, which results in fewer base station requirements to cover an area.

3. Increase in Capacity. Smart antennas enable reduction in co-channel interference which leads to increase in the frequency reuse factor i.e. more users can use the same frequency spectrum at the same time bringing about tremendous increase in capacity.

4. Reduction in Transmitted Power: Smart antennas radiate energy in a particular desired direction. Therefore, less power is required for radiation at the base station. Reduction in transmitted power also implies reduction in interference towards other users.

5. Reduction in Handoff: Capacity of a cellular network can be increased by dividing the congested cells in to micro cells which further increase the frequency reuse factor this results in frequent handoffs as the cell size is smaller. Using smart antennas at the base station, there is no need to split the cells as the capacity is increased by using independent spot beams.

\section{Conclusion:}

In this paper we have presented a comparative analysis of different types of feeding techniques of microstrip patch antennas, which represent their uses as per different applications. Along with this we propose smart antenna concept which is a new paradigm in the field of mobile communication. The smart antennas are applicable to all major wireless protocols and standards. They immensely modify wireless transmission efficiency.

Smart antennas offer several advantages over omnidirectional or sector antennas. These include increased coverage through range extension, increased capacity achieved through interference reduction, and mitigation of multipath fading and inter symbol interference. As Smart antennas technology becomes cheaper it is likely to be used in all other wireless devices. Along with this, still there are several challenges to be resolved before the full potential of smart antennas can be realized.

\section{References:}

[1]. James J.and P.S. Hall (Eds), "Handbook of microstrip antenna", Peter Peregrinus, London, UK, 1989.

[2]. Indrasen Singh, Dr. V.S. Tripathi, "Microstrip patch antenna and its applications: a Survey ", Int. J. Comp. Tech. Appl., Vol. 2 (5), 1595-1599, ISSN: 2229-6093.

[3]. Liton Chandra Paul, Nahid Sultan,"Design, Simulation And Performance Analysis Of A Line Feed Rectangular Micro-Strip Patch Antenna “, Int.J.of Engg. Sci. \& Emerg. Tech., Feb. 2013. Vol. 4( 2), pp: 117-126 ,ISSN: 2231 - 6604.

[4]. Amit Kumar, Jaspreet Kaur, Rajinder Singh "Performance Analysis of Different Feeding Techniques," Int. J. of Emerg. Tech. and Ad. Engg.” , Vol. 3( 3), March 2013, ISSN 2250-2459, ISO 9001:2008 Certified Journal.

[5]. RK Jain, Sumit Katiyar, NK Agrawal,"Smart Antenna for Cellular Mobile Communication "VSRD-IJEECE, Vol. 1 (9), 2011, 530541

[6]. Murali Kiruba "Smart Antennas for Wireless Mobile Communication" IT toolbox Wireless- 15592, 22 Oct 2004.

[7]. Martin Cooper, Marc Goldburg,"Intelligent Antennas: Spatial Division Multiple Access” Annual Review of Communications, 1996.

[8]. Joseph Shapira, "Microcell Engineering in CDMA cellular Networks" IEEE Transactions on Vehicular Technology, Vol. 43, No. 4, Nov 1994.

[9]. Hala Elsadek,"Microstrip Antennas for mobile Wireless communication system", Research Institute, Microstrip Department, Cario, Egypt

[10]. Rameshwar Kawitkar, "Issues in Deploying Smart Antennas in Mobile Radio Networks, " proceedings of World Academy of Science, Engg.and Tech. Vol. 31 July 2008, ISSN 2070-3740.

[11]. Ch. Santhi Rani, Dr. P V Subbaiah, Dr. K Chennakesava reddy, "Smart Antenna Algorithms for WCDMA Mobile Communication Systems" Int. J. of Comp. Sci.and Network Security, Vol. 8 No. 7, July 2008.

[12]. K. Praveen Kumar, K. Sanjeeva Rao, T. Sumanth, N. Mohana Rao, R. Anil Kumar, Y.Harish, "Effect of Feeding Techniques on the Radiation Characteristics of Patch Antenna: Design and Analysis," Int.J. of Ad.R.in Comp. and Comm.Engg.Vol. 2, Issue 2, February 2013"

[13]. Hui Liu and Guanghan Xu. Multiuser blind channel estimation and spatial channel pre-equalization. IEEE Proceedings, 1995.

[14]. C. Farsakh and J. A. Nossek.,"Application of space division multiple access to mobile radio",Proceedings PIMRC, pages 736-739, 1994. 